International Journal of Pure and Applied Mathematics

Volume 109 No. 2 2016, 443-449

ISSN: 1311-8080 (printed version); ISSN: 1314-3395 (on-line version)

url: http://www.ijpam.eu

doi: 10.12732 ijpam.v109i2.17

\title{
SIGNAL DETECTION IN SAR AGAINST THE CLUTTER REFLECTIONS BACKGROUND
}

\author{
Leonid G. Dorosinskiy \\ Ural Federal University Named after the First \\ President of Russia B.N. Yeltsin \\ 620002, Ekaterinburg, RUSSIAN FEDERATION
}

\begin{abstract}
This paper presents the algorithm of multiple-unit target acquisition against the structurally similar interference and analysis method of its efficiency. Synthesized aperture antenna pattern in some practical situations along with the valid signal reflected from the multiple-unit target has rather powerful interfering signals produced by the reflections from the interfering objects. The algorithm of multiple-unit target acquisition against the multipleunit interference and analysis method of its efficiency have been presented.
\end{abstract}

AMS Subject Classification: 11A05, 60G35, 65D15, 93E10, 94A12

Key Words: algorithm, detection, target, interference

\section{Introduction}

The forming problem of the optimal algorithm of signal detection in the radio detector with synthesized aperture with the presence of the clutter reflections from the local objects and the development of the efficiency estimation method of such detection is the main problem in the designing and building of the remote sensing systems of earth and water surfaces by the air and satellite observational platforms with synthesized aperture.

Received: Jule 20, 2016

Published: September 10, 2016 (c) 2016 Academic Publications, Ltd.

url: www.acadpubl.eu 


\section{Concept Headings}

Devoted to the problems of signal processing within the radar station with the synthesized aperture (SAR) papers [1], [2], [3] pay great attention to the research of the detection algorithms under interferences caused by the reflection from the underlying surface and noises. SAR antenna pattern in some practical situations along with the valid signal reflected from the multiple-unit target has rather powerful interfering signals produced by the reflections from the interfering objects. Therefore, in these cases the processing algorithm should be formed accounting both the distribution target character and the interference presence. The determination of the main principles of algorithm forming and the analysis methods present the content of this paper.

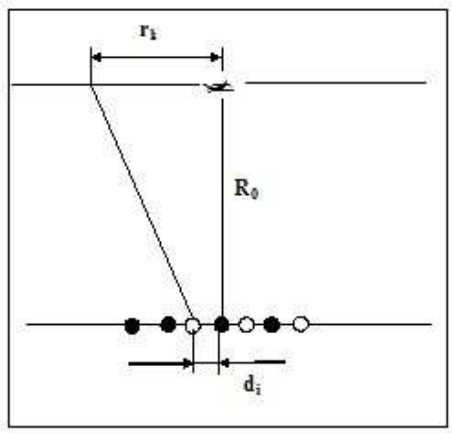

Figure 1: Task geometry

Suppose, the side-looking radar moves along the linear path. The range resolution cell has the valid and interfering signals formed by the separate reflectors which are distant at $d_{i}^{c}(i=\overline{1, n})$ and $d_{i}^{n}(i, \overline{1, N})$ from the coordinate origin and $\mathrm{n}$ and $N$ - the numbers of signal and interfering reflectors respectively (Figure 1). Under the discrete time processing the vector of the observed data is presented in the following form:

$$
Y=\beta_{c} A_{c}+\beta_{n} A_{n}+N_{m}
$$

where

$$
\beta_{c}=\left\|X\left(d_{1}^{c}\right), \ldots \ldots, X\left(d_{n}^{c}\right)\right\|
$$

$-M \times n$ matrix consisting of vectors

$$
X\left(d_{i}^{c}\right)=\left\|\exp \left(j \frac{2 \pi}{\lambda R_{0}} r_{k}^{2}-j \frac{4 \pi}{\lambda R_{0}} d_{i}^{c} r_{k}\right)\right\|,
$$


setting the phase signal distribution reflected from $i$-target element on the points of synthesized aperture with the coordinates $r_{k}, k=\overline{1, M}(\lambda$ - wavelength); $A_{c}$ and $A_{n}-(n \times 1)$ and $(N \times 1)$ vectors of complex signal (interference) amplitude which are normal random variables with zero mathematical expectations and dispersion $\sigma_{c i}^{2}$ and $\sigma_{n i}^{2}$ respectively; matrix $\beta_{n}$ is determined similarly to (2) and (3), $N_{m}$ - the complex amplitude vector of gaussian noise.

Recording the observed data in the form (1), the quadric form is a sufficient statistics for the detection of the valid signal

$$
\alpha=Y^{* T} \theta Y
$$

where $\theta=R_{n}^{-1}-R_{S P}^{-1}-$ the processing weight function,

$$
\begin{aligned}
& R_{s p}=\beta_{c} Q_{c} \beta_{c}^{* T}+R_{n} \\
& R_{n}=\beta_{n} Q_{n} \beta_{n}^{* T}+R_{m}
\end{aligned}
$$

the correlation matrixes of vector (1) with or without the valid signal

$$
\begin{gathered}
Q_{c}=\operatorname{diag}\left(\sigma_{c i}^{2}, \ldots \ldots, \sigma_{c n}^{2}\right) \\
Q_{n}=\operatorname{diag}\left(\sigma_{n 1}^{2}, \ldots \ldots, \sigma_{n N}^{2}\right) \\
R_{m}=\sigma_{m}^{2} E
\end{gathered}
$$

where $*$ - complex conjugation, $T$ - transpose sign, $E$ - identity matrix, next count the noise dispersion without the loss of generality $\sigma_{m}^{2}=1$.

Using Woodbury formula for the determination of the optimal weight function write the equation of the sufficient statistics in the following form

$$
\alpha=Z^{* T} P Z
$$

where

$$
\begin{gathered}
P=\left(E+Q_{c} \beta_{c}^{* T} R_{n}^{-1} \beta_{c}\right)^{-1} Q_{c}, \\
R_{n}^{-1}=E-\beta_{n}\left(E+Q_{n} \beta_{n}^{* T} \beta_{n}\right)^{-1} Q_{n} \beta_{n}^{* T} \\
Z=Y^{T} R_{n}^{-1} \beta_{c}^{*}=Y^{T} X^{*}\left(d_{i}^{c}\right)-\sum_{l=1}^{N} \chi_{l i} Y^{T} X^{*}\left(d_{l}^{n}\right. \\
\chi_{l i}=\sum_{t=1}^{n} p_{l t} X^{T}\left(d_{t}^{n}\right) X^{*}\left(d_{i}^{n}\right)
\end{gathered}
$$

$p_{l t}$ - the matrix element 11 . 


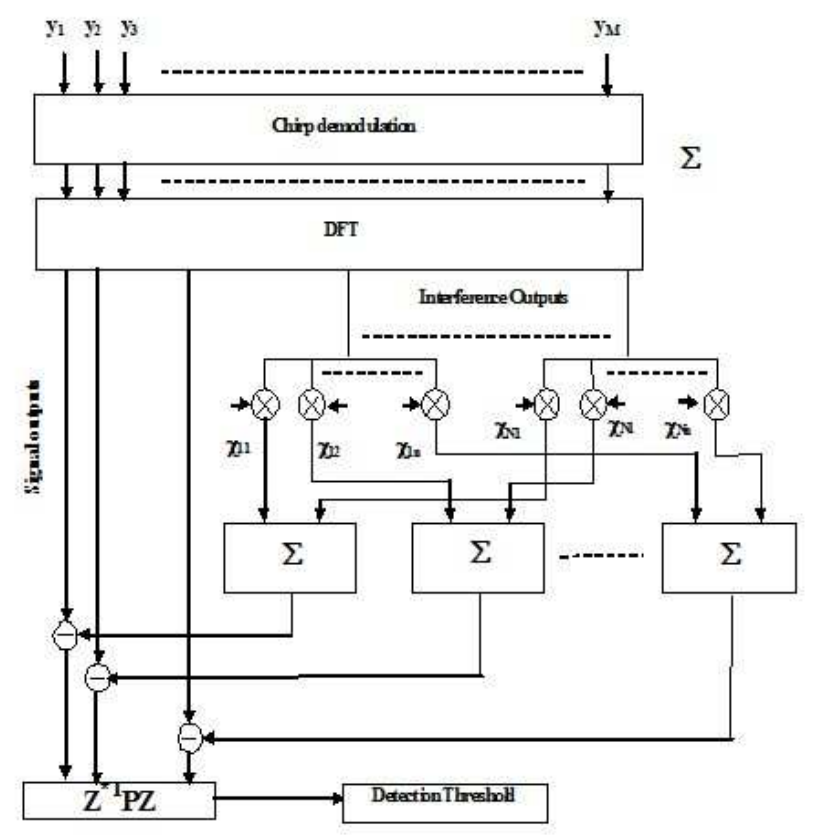

Figure 2: Optimal algorithm flowchart

The schematic structure with the optimal algorithm (10) is shown on Fig. 2. The main functional operation in (13):

$$
Y^{T} X^{*}\left(d_{i}\right)=\sum_{k=1}^{M} y_{k} \exp \left(-j \frac{2 \pi}{\lambda R_{0}} r_{k}^{2}+\frac{4 \pi}{\lambda R_{0}} d_{i} r_{k}\right),
$$

is chirp demodulation and discrete Fourier transform (DFT) estimated for the spatial frequencies $\left(2 / \lambda R_{0}\right) d_{i}$, corresponding to all the elements of target (interferences).

The spectral components which correspond to the interferences are estimated accounting the penetration of interferences into the signal channels and subtracted from the complex amplitudes of signal spatial harmonics. The quadric form of the output voltage of signal channels allows accounting of signal interpenetration from different target elements into the adjacent channels.

The implementation of optimal processing (10)-(14) substantially increases the detection efficiency of small-size objects against the powerful interfering reflections. Therefore, with the interference (the interference-noise power ratio $M \sigma_{n}^{2} / \sigma_{m}^{2}=10^{3}$ ), the angular direction which matches with the main leaf border of synthesized antenna pattern in the half power level, the gain of the signal-tonoise ratio with the optimal processing is more than $23 \mathrm{~dB}$ in comparison with 
the agreed processing usually used in SAR. This case the expected measurement accuracy of interference coordinates does not exceed $5-10 \%$ width of the main antenna pattern lobe.

The relative gain of the optimal processing in comparison with the traditional one in PSA does not allow the estimation of the absolute values of the detection characteristics with multiple-unit sources of signals and interferences. On the other hand, the exact calculation of these characteristics is connected with the significant calculating difficulties caused in the determination and integration of distributed statistics (10). Therefore, the efficiency estimation of the considered algorithm uses the method based on Chernoff bound [4], [5] according to which the probabilities of correct detection and false alarm are counted on the formulae:

$$
\begin{gathered}
P_{D}=1-\exp \left\{\gamma\left[\nu(s)+(1-s) \dot{\nu}(s)+0,5(1-s)^{2} \ddot{\nu}(s)\right]\right\} \times \\
\times \operatorname{erfc}[(1-s) \sqrt{\gamma \nu(\ddot{s})}], \\
P_{F}=\exp \left\{\gamma\left[\nu(s)-s \dot{\nu}(s)+0,5 s^{2} \ddot{\nu}(s)\right]\right\} \times \operatorname{erfc}[(1-s) \sqrt{\gamma \nu(\ddot{s})}]
\end{gathered}
$$

where

$$
\nu(s)=\ln \int_{-\infty}^{\infty} \ldots \int_{-\infty}^{\infty}[P(Y / c+n)]^{s}[P(Y / n)]^{1-s} d Y ;
$$

$\dot{\nu}(s)$ and $\ddot{\nu}(s)$ - the first and second derivatives of (18), $s=0 \div 1$ - the dummy argument, $\gamma$ - the number of independent tests (for PSA - the number of used frequencies with multi frequency probing or of the number of non coherent summed synthesized images with partly coherent PSA working order $), P(Y / c+$ $n), P(Y / c+n)$ - the probabilities density of the observed vector with the presence or absence of the valid signal.

According to the case of the present paper the formula (18) has the following form

$$
\nu(s)=\sum_{i=1}^{M} \ln \frac{\lambda_{i}^{1-s}}{s+\lambda_{i}(1-s)}
$$

where $\lambda_{I}$ - the intrinsic matrix values $\hat{\Phi}_{0}^{T} R_{s p} \hat{\Phi}_{0}^{*}$

$$
\hat{\Phi}=\Delta_{0}^{1 / 2} \Phi_{0}, \Delta_{0}=\operatorname{diag}\left(\lambda_{01}, \ldots, \lambda_{0 M}\right)
$$

$\lambda_{0 i}, \Phi_{0 i}$ - the intrinsic values and vectors of the matrix (6)

The characteristics of multiple-unit target $(n=5)$ against the multiple-unit interference $(\mathrm{N}=5)$ are calculated on the formulae (16) and (17) accounting 
(19). The every of four shown detection characteristics (Figure 3) corresponds to the interfering situation on Figure 4, where the mutual angular position of target and interference are indicated in the shares of normalized coordinates

$$
\mu=\frac{L}{\lambda} \sin \theta,
$$

where $L$ - the length of coherent integration, $\lambda$ - the wavelength, $\theta=\operatorname{arctg}\left(d / R_{0}\right)$ - the angular direction on the signal source or interference. There are 8 coherent summed impulses and 21 non-coherent summed sectors. The signal-tointerference ratio is along $X$-direction $q \sigma_{c}^{2} / \sigma_{n}^{2}$.

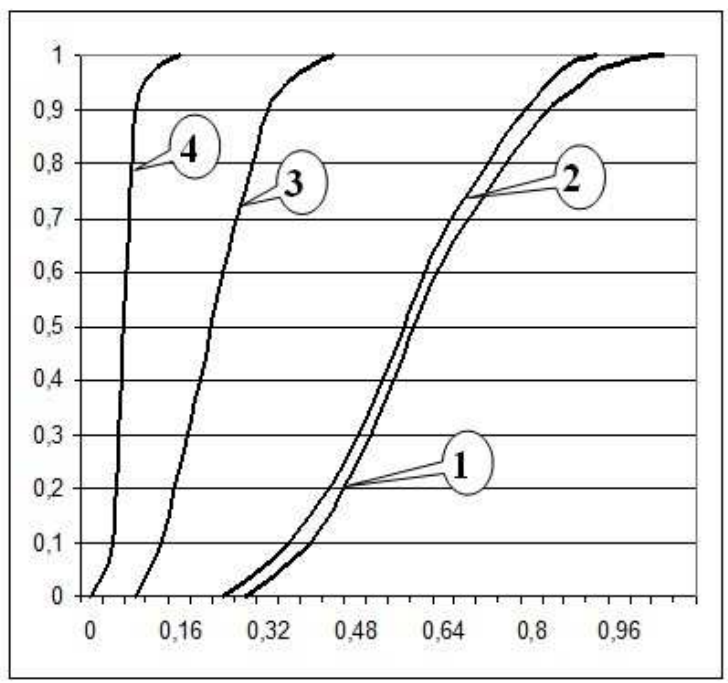

Figure 3: Detection characteristics

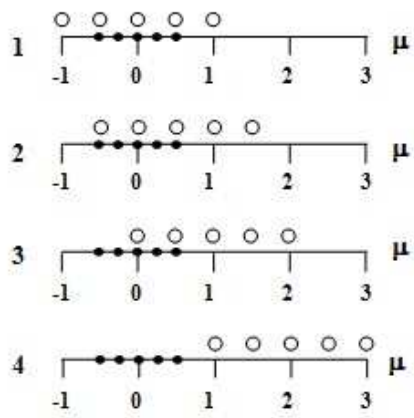

Figure 4: Signal and noise source location 


\section{Conclusion}

The presented curves demonstrate that even with the target or interference within the main leaf of synthesized antenna pattern $(q<1)$ the probability of correct decision $P D>0,9$ with $q<1$. The placement of interference source elements within the first two $D P$ leaves (curve 4) $P D>0,9$ with $q<0,1$, that testifies the high efficiency of optimal processing.

\section{References}

[1] L.G. Dorosinskiy, Radar Signals Class Recognition Algorithm Synthesis Applied and Fundamental Studies, Proceedings of the $4^{\text {st }}$ International Academic Conference, Vol. 1, St Louis, Missouri USA (2013).

[2] G.S. Kondratenkov, V.A. Potekhin, A.P. Reutov, Y.A. Feoktistov, Earth observation radar stations, Moscow, Radio and Telecommunications (1983).

[3] L.G. Dorosinskiy, Synthesis of Signal Flow Group Parameter Optimal Indicator, Proceedings of the $4^{\text {st }}$ International Academic Conference, Vol. 1, St Louis, Missouri USA (2013).

[4] L.G. Dorosinskiy, The research of the distributed objects' radar image recognition algorithms, Applied and Fundamental Studies, Proceedings of the $2^{\text {st }}$ International Academic Conference, Vol. 1, St Louis, Missouri USA (2013).

[5] G. van Treese, Detection, evaluation and modulation theory, Moscow, Soviet Radio, Vol 1 (1979). 
